\title{
Multidisciplinary Approach to Prevent Limb Amputation in Diabetic Patients
}

\author{
Amol Gupta ${ }^{\mathrm{a}, \mathrm{b}}$, Vinod Kumar
}

\begin{abstract}
Diabetes mellitus is a complex disease with clinical sequelae including cardiovascular disease, renal failure, extremity complications, and death. Lower limb amputation is the most serious morbidity and consequence for diabetic patients due to its associated burden on quality of life and economic implications. Limb amputation is caused by a series of complications involving multiple organ systems. We believe that a multidisciplinary diabetic care team, consisting of vascular specialists, wound care specialists, and cardiologists, will facilitate in systematically addressing each complication leading to amputation. Diabetic patients have an increased risk of developing cardiovascular diseases, resulting in the expanding involvement and importance of interventional cardiologists on these multidisciplinary diabetic care teams. Several national and global institutions have successfully demonstrated the multidisciplinary approach to facilitate efficient and timely care, and most importantly, reduce the rates of diabetes-related limb amputation. The Heart, Vascular and Leg Center coordinates vascular specialty care and provides dedicated patient management in order to increase treatment quality, patient literacy, and medication adherence to decrease lower extremity amputation rates.
\end{abstract}

Keywords: Diabetes; Limb amputation; Multidisciplinary approach; Peripheral arterial disease

\section{Alarming Rates of Diabetes-Related Amputa- tion}

The global prevalence of diabetes mellitus has risen over the past several decades, with prevalence rates estimated at $8.8 \%$ of the world population. Recent estimates project that this rate will continue to rise, reaching a global prevalence of over $10 \%$ by 2035 [1]. Diabetes currently accounts for $9 \%$ of mortality worldwide [2], with cardiovascular disease (CVD) being the primary causal factor leading to death in diabetic patients.

Manuscript submitted September 23, 2019, accepted October 4, 2019

${ }^{a}$ Cardiology, Heart, Vascular \& Leg Center, Bakersfield, CA, USA

${ }^{\mathrm{b} C}$ Corresponding Author: Amol Gupta, Heart, Vascular \& Leg Center, 5020

Commerce Drive, Bakersfield, CA 93309, USA. Email: amol@vippllc.com

doi: https://doi.org/10.14740/jem615
CVD in diabetic patients can lead to several downstream morbidities, perhaps the most severe of which is lower extremity limb amputation due to its association with poor quality of life and low survival rates [3].

Limb amputation in diabetic patients is performed approximately every $30 \mathrm{~s}$ globally, equating to a loss of over 2,500 limbs every day [4]. Limb amputation lowers quality of life and places severe economic burden on patients and healthcare systems. For this reason, amputation connotes a treatment failure rather than a treatment option. Multiple studies have demonstrated significant deterioration of physical and mental health post-limb amputation [5-9]. Hayes et al evaluated the quality of life of diabetes patients with various complications via self-reported questionnaires, and found that patients who underwent limb amputation had the largest decrement in physical quality of life compared to other complications such as stroke, blindness, renal failure, and heart failure [6]. Similarly, a recent systematic review of the literature by Davie-Smith et al demonstrated a high prevalence of depressive symptoms and feelings of social isolation amongst diabetic patients with major lower limb amputation, especially those over age 65 [9].

\section{The Financial Costs of Limb Amputation}

In addition to lowering health and lifestyle quality, limb amputations are financially taxing to both patients and healthcare systems [10-14]. Medical care costs for minor and major amputations, as reported by Jindeel and Narahara, were $\$ 45,513$ and $\$ 82,657$, respectively, amongst diabetic patients, which was approximately 1.3 -fold higher than the cost for treating non-diabetic amputations [12]. Franklin et al further corroborated these high costs, reporting that the mean cost of lower limb amputation per diabetic patient treated by the Veterans Health Administration (VHA) approximated \$60,647 in 2010, amounting to an overall economic burden of $\$ 206$ million [14].

\section{Solution to the Amputation Endemic Amongst Diabetic Patients}

A multidisciplinary approach to diabetic care has been found to optimize treatment for both the patient and the wound in several recent reports [15-18]. In a retrospective review, Wang et al found a decrease in lower extremity amputations associated with implementation of a multidisciplinary approach to diabe- 
tes treatment, coordinated by an endocrinologist and podiatrist [15]. Similar decreases in amputation rates and ulcer healing times were reported by Everett and Mathioudakis after introducing a multidisciplinary approach [17]. In addition to these findings, Oliver and Mutluoglu noted that consults with internal medicine, podiatry, endocrinology, vascular surgery, and infectious disease are key integrations for a multidisciplinary team, and can collectively aid in promoting patient education and prevention of foot ulcers ultimately leading to amputation [16]. Moreover, the World Health Organization and the International Diabetes Foundation reported that approximately $85 \%$ of diabetes-related lower extremity limb amputations can be avoided with appropriate preventive and specialist care [15, 16, 19-23].

\section{A One-Stop Shop: Why the Multidisciplinary Ap- proach Works}

Diabetes-related complications leading to limb amputation are multifactorial, involving neuropathy of peripheral vasculature, ulceration, presence (or absence) of peripheral arterial disease (PAD), and infection [2, 24]. Thus, diabetic foot problems involve pathology of various systems, requiring the need of multiple specialists to address each component. The multidisciplinary team consists of professionals from three primary disciplines: vascular specialists, cardiology, and wound care.

Vascular diseases are a primary causal factor leading to lower extremity amputation, accounting for $82 \%$ of amputations amongst diabetic patients with foot problems. Surprisingly, a 2001 study by Allie et al revealed that only $49 \%$ of those receiving amputations had any diagnostic vascular evaluation prior to amputation [25]. Even over a decade later, Goodney et al found that of 20,464 Medicare patients who underwent major amputation for critical limb ischemia, 71\% had either no revascularization or only one diagnostic procedure in the year immediately preceding a major amputation [26]. Moreover, amputation was the first method of treatment used in $67 \%$ of these patients. Quilici et al found that using nonsurgical treatments prior to admission decreased amputation rates by $63 \%$ [19]. With careful monitoring and care by vascular specialists, diabetic patients can receive earlier diagnosis and intervention for commonly associated cardiovascular complications such as atherosclerosis.

Wound care specialists serve an important role in the multidisciplinary diabetic care team. Ulceration and associated infection occurs in $15 \%$ of diabetic patients; lack of early intervention and proper care often leads to major or minor amputations [27]. Of note, nearly $85 \%$ of amputations in diabetic foot patients are preceded by diabetic foot ulceration [28]. Thus, wound care specialists are a critical member of the diabetic care team and facilitate prevention and management of wound infections.

CVD is the leading cause of morbidity and mortality amongst diabetic patients. In fact, $35 \%$ of diabetic patients with PAD will die within 5 years from coronary artery disease (CAD) or CVD. Diabetes is a frequently seen condition by cardiologists, who work closely with diabetic patients to ensure glycemic control, blood pressure management, cholesterol and lipid level maintenance, and management of other risk factors that will prevent major complications (i.e. myocardial infarction, artherosclerotic heart disease) [29]. Together, the cardiologist and vascular specialist work to improve functional status and quality of life of the diabetic patient by identifying and treating their systemic atherosclerosis, while the wound care specialist manages the patient's ulcer wounds to prevent infection spread. As a result, the multidisciplinary team can collectively and systematically manage all components of diabetes to aid in limb preservation. We have seen the role of the cardiologist expand over the last two decades, discussed in detail below.

In addition, two of the main factors related to lower extremity limb amputation in diabetic populations are non-compliance with outpatient medication and previous outpatient treatment for ulcers [19]. Socioeconomic factors and social determinants of health may play a role in a patient's ability to access appropriate clinical care and remain adherent with medications, and thus are important factors in subsequent amputation and clinical outcomes. Coordinating patient access to care via a multidisciplinary team may potentially improve patient compliance and ultimately reduce subsequent costs and poor clinical outcomes resulting from lower extremity amputation.

Many institutions have recognized the need for an integrated, multidisciplinary approach for diabetes care and have implemented initiatives to mitigate diabetes-related amputation through specialized diabetic foot care clinics and early intervention programs and have demonstrated drastically improved clinical outcomes, discussed in detail below.

\section{Denver Health Study}

In 2004, the Denver Health Medical Center established a limbsalvage program to address the specialized needs associated with caring for diabetic foot complications. A multidisciplinary team was formed consisting of podiatrists, orthopedic and general surgeons, primary care physicians, and specialists in infectious disease and interventional cardiology. The process of care through this program involved scheduled foot exams, patient education, vascular education, footwear and wound care, and surgery, when necessary, performed by podiatrists. A 10 -year retrospective study of 451 patients treated in the Denver Health limb-salvage program demonstrated that prior to the program, limbs were lost in $36.3 \%$ of cases, compared to only $11 \%$ between 2006 and 2011 upon establishing the multidisciplinary approach [30].

\section{Brazilian "Diabetic Save the Foot Project"}

Using a multidisciplinary approach in treating diabetic foot conditions has been globally recognized as a beneficial strategy to prevent limb amputation as early as the 1990s. In 1992, the Brazilian government launched the "Diabetic Save the Foot Project", aimed at managing diabetic foot problems, a main reason for amputation in developing countries. The program was designed to circumvent barriers to healthcare access by foot clinics throughout the country, by improving preventative education programs for patients, and increasing awareness of diabetic foot complications amongst medical staff through col- 
laborative interdisciplinary efforts. Due to the shortage of podiatrists and chiropodists in the country, diagnostics and treatment were performed by a multidisciplinary team involving nurses, primary care physicians, vascular surgeons, and endocrinologists. Other specialists, such as plastic surgeons, were available to patients upon referral. All staff received training of basic podiatry procedures and strategies to educate patients on diabetic foot condition management. A retrospective analysis of the data from this project demonstrated a steady decline in amputation rates between 1992 and 2000, achieving a notable overall decrease of $77.8 \%$ [31].

\section{District Hospital, Ipswich, United Kingdom}

The Ipswich Hospital, a district general hospital in the United Kingdom, performed a study between 1995 and 2005 to evaluate the changes in diabetic lower extremity amputation rates following the implementation of a multidisciplinary foot team. The data were collected prospectively during this period by a diabetes specialist nurse or podiatrist. Study outcomes revealed a significant decrease in the number of total and major amputations. The incidences of total, major, and minor amputations were reduced by $70.0 \%, 81.8 \%$, and $21.1 \%$, respectively, during the 11-year period [32]. The improved foot care outcomes were attributed to the collaborative efforts of the vascular, radiological, and microbiological services of the multidisciplinary team.

\section{VHA}

The VHA is the largest national database of medical records in the United States. Previous studies have utilized combined data from the VHA and Medicare database to develop risk prediction models for major and minor amputations in diabetic patients [33-35]. Between 2000 and 2004, the Department of Veterans Affairs conducted a retrospective administrative data analysis of VHA clinic users with diabetes to assess the trends in lower extremity amputations. The study population was defined as patients with diabetes without prior amputation and ranged between 405,580 in 2000 to 739,377 in 2004. The trend in amputation rates was calculated per 1,000 patients for minor (toe, transmetatarsal, and distal transtibial) and major (transtibial and transfemoral) amputations. Over the 5-year period, minor amputations were reduced by $33 \%$ and a $36 \%$ decrease was observed for major amputations [36]. Above knee amputations were reduced by $49 \%$. It was speculated that the significant decreases in lower extremity amputations were largely a result of the foot risk screening and referral program mandated by the VHA in the late 1990s [37].

\section{Expanding Role of the Cardiologist}

The role of the cardiologist has changed dramatically as clinical problems that are vascular in origin become more common. Over the last 20 years, cardiologists have become more involved in the treatment of peripheral vascular diseases [38]. The fundamental competencies required to diagnose and treat vascular diseases are explicitly detailed in a recent Core Cardiovascular Training Statement (COCATS) [39]. Additionally, interventional and imaging procedures in the peripheral vascular system have become important components of a cardiologist's practice and curriculum in recent years $[38,40]$. The past several decades have also shown tremendous growth in the proportion of endovascular interventions performed by cardiologists. Goodney et al found that between 1996 and 2006, the number of endovascular procedures performed by cardiologists increased from 29 to 170 interventions per 100,000 Medicare beneficiaries, respectively, compared to an increase from 12 to 162 endovascular interventions per 100,000 Medicare beneficiaries by specialized vascular surgeons [41]. Furthermore, the authors showed that the proportion of all endovascular procedures performed by cardiologists increased from $23 \%$ to $41 \%$ between 1996 and 2000, clearly demonstrating the expanding role of interventional cardiologists for the treatment of vascular diseases [41].

Individuals with diabetes have an increased risk of developing a CVD [42]. CVDs are currently the number one cause of death worldwide, with almost 18 million global deaths reported in 2016 [43]. CAD and PAD are chronic progressive CVDs that share similar risk factors and pathogenesis [38]. Patients with PAD or CAD are treated similarly, but are also at risk of secondary thrombotic events and concomitant diseases in other vascular districts [44-46]. Cardiologists are often the first physicians to see patients with CAD or PAD, and are responsible for managing multiple aspects of these vascular disorders. Given their early access to patients with these disorders, cardiologists must use this opportunity to screen patients. Given their unique training as interventionalists, cardiologists are also equipped to perform endovascular procedures. Because these CVDs often engage several disciplines, cardiologists serve as an increasingly important, unifying member on multidisciplinary treatment team.

A number of studies demonstrate the growing success when interventional cardiologists are the operating physicians in endovascular procedures. Khanolkar and Ephrem retrospectively analyzed 24 patients that underwent endovascular reconstruction of popliteal and/or infrapopliteal arteries for critical limb ischemia between 2010 and 2014 [47]. Limb salvage was achieved in $97 \%$ (33/34) of their patients and successful vessel recanalization was achieved in $71 \%(24 / 34)$. Their success with endovascular procedures is reflected by the increasing number of interventional cardiologists participating in endovascular trials for critical limb ischemia. Of the 865 physicians participating in the Best Endovascular vs. Best Surgical Therapy for Patients with Critical Limb Ischemia (BEST-CLI) trial, 128 (15\%) are interventional cardiologists [48]. The involvement of these skilled interventionalists will continue to expand as less-invasive endovascular therapies are used to treat vascular diseases.

\section{Heart, Vascular and Leg Center (HVLC) Experi- ence}

The HVLC provides multidisciplinary care to diabetes pa- 


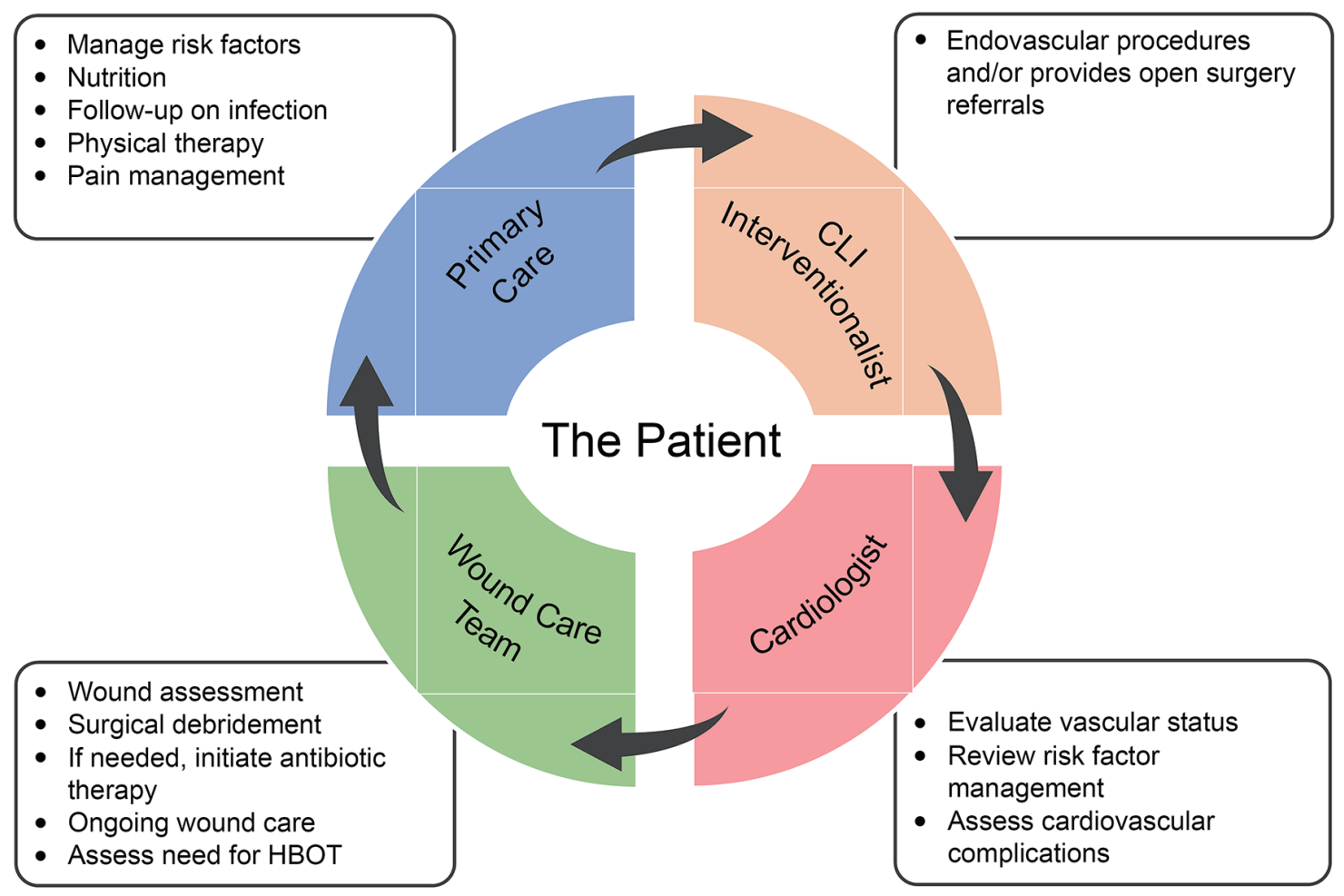

Figure 1. Diagram describing the roles of each member on our multidisciplinary, patient-centered team to prevent limb amputation.

tients by bringing cardiology, podiatry, vascular, and wound care specialties into a single treatment center in order to use a "whole person" approach to amputation prevention (Fig. 1). Bringing multifaceted patient care into a single treatment center allows for an integrated system and approach to treatment that promotes peer communication between physicians as well as communication between physicians and patients. Through increasing both peer and patient communication channels, we decrease care access challenges for patients and provide dedicated patient management to coordinate care at a higher level than through individual specializations, allowing for increased tracking of patient care.

Once a patient is referred to the HVLC, the wound care team assesses their non-healing wound and refers the patient to assessment by a cardiovascular specialist. A cardiologist will evaluate their vascular status, ongoing risk factors, and need for testing, angiography, or lower extremity intervention. An endovascular specialist will then communicate with the multidisciplinary team to ensure limb revascularization if treatment is required. Follow-up assessments will take place $24 \mathrm{~h}$ postrevascularization, with weekly assessments by a primary care physician, and ongoing cardiovascular assessments for the rest of the year (Fig. 2).

The multidisciplinary approach has enhanced several key areas of patient care at the HVLC facility. First, we have seen improvements in patient compliance. Consistent visits with the same provider and dedicated staff member have also resulted in a decrease in missed appointments. Second, the number of hospital visits has decreased tremendously, as infections are diag- nosed and treated earlier due to weekly wound checks. Finally, monthly meetings enable us to develop protocols, address any issues, and improve communication between multidisciplinary providers working on the same EMR. We anticipate continued refinements and growth at the facility to ensure that patients are provided with the best possible multidisciplinary care.

\section{Conclusion}

Coordinated care improves patient compliance and physician communication, enhancing treatment management as coordinators track patient progress. The combined efforts of specialists from different disciplines will provide diabetic patients with the best possible care and can reduce overall limb amputation rates. Interventional cardiologists have become increasingly important providers of care for diabetic patients on multidisciplinary teams; the role of cardiologist will continue to expand for diabetic patients as diseases that are vascular in origin become more common. Multidisciplinary approaches to diabetic treatment ultimately provide patients with a better understanding of their treatment and improves medication adherence, lower extremity amputation rates, and improve clinical outcomes.

\section{Acknowledgments}

The authors acknowledge Superior Medical Experts for editing assistance. 


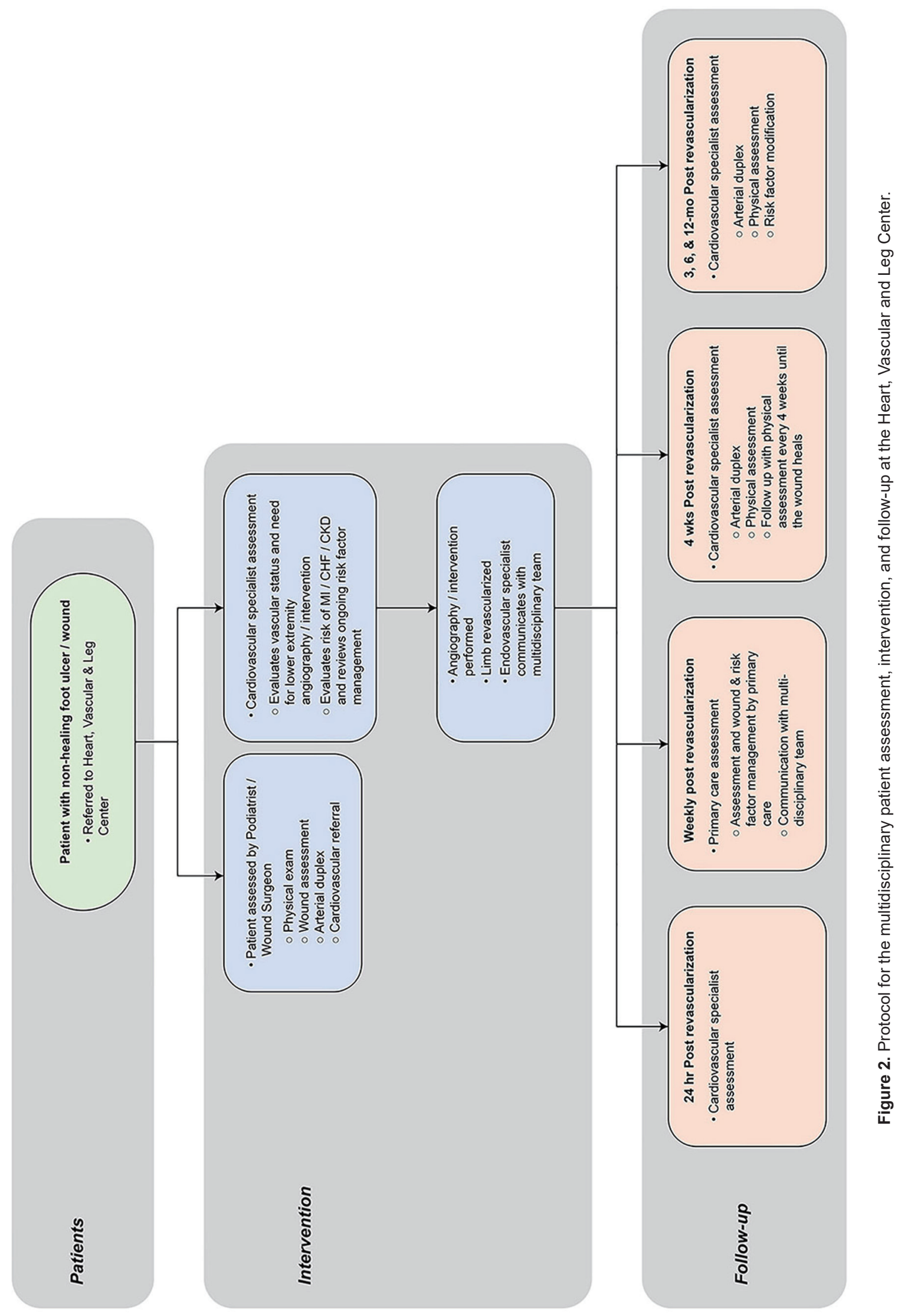




\section{Financial Disclosure}

None to declare.

\section{Conflict of Interest}

The author reports no conflict of interest in this work.

\section{Author Contributions}

AG contributed to the conception and design of the work, literature search, data analysis for the work, and drafting the manuscript; VK contributed to the conception and design of the work and critically revised the manuscript.

\section{References}

1. Baena-Diez JM, Penafiel J, Subirana I, Ramos R, Elosua R, Marin-Ibanez A, Guembe MJ, et al. Risk of cause-specific death in individuals with diabetes: a competing risks analysis. Diabetes Care. 2016;39(11):1987-1995.

2. Rodrigues BT, Vangaveti VN, Malabu UH. Prevalence and risk factors for diabetic lower limb amputation: a clinic-based case control study. J Diabetes Res. 2016;2016:5941957.

3. Shin JY, Roh SG, Sharaf B, Lee NH. Risk of major limb amputation in diabetic foot ulcer and accompanying disease: A meta-analysis. J Plast Reconstr Aesthet Surg. 2017;70(12):1681-1688.

4. Bharara M, Mills JL, Suresh K, Rilo HL, Armstrong DG. Diabetes and landmine-related amputations: a call to arms to save limbs. Int Wound J. 2009;6(1):2-3.

5. Bosic-Zivanovic D, Medic-Stojanoska M, Kovacev-Zavisic B. [The quality of life in patients with diabetes mellitus type 2]. Vojnosanit Pregl. 2012;69(10):858-863.

6. Hayes A, Arima H, Woodward M, Chalmers J, Poulter N, Hamet P, Clarke P. Changes in quality of life associated with complications of diabetes: results from the ADVANCE study. Value Health. 2016;19(1):36-41.

7. Pedras S, Carvalho R, Pereira MG. Quality of life in portuguese patients with diabetic foot ulcer before and after an amputation surgery. Int J Behav Med. 2016;23(6):714721.

8. Alves Costa MS, Pereira MG. Predictors and moderators of quality of life in caregivers of amputee patients by type 2 diabetes. Scand J Caring Sci. 2018;32(2):933-942.

9. Davie-Smith F, Coulter E, Kennon B, Wyke S, Paul L. Factors influencing quality of life following lower limb amputation for peripheral arterial occlusive disease: A systematic review of the literature. Prosthet Orthot Int. 2017;41(6):537-547.

10. Foster D, Lauver LS. When a diabetic foot ulcer results in amputation: a qualitative study of the lived experience of 15 patients. Ostomy Wound Manage. 2014;60(11):16-22.

11. Joret MO, Dean A, Cao C, Stewart J, Bhamidipaty V. The financial burden of surgical and endovascular treatment of diabetic foot wounds. J Vasc Surg. 2016;64(3):648-655.

12. Jindeel A, Narahara KA. Nontraumatic amputation: incidence and cost analysis. Int J Low Extrem Wounds. 2012;11(3):177-179.

13. Schaper NC, Apelqvist J, Bakker K. Reducing lower leg amputations in diabetes: a challenge for patients, healthcare providers and the healthcare system. Diabetologia. 2012;55(7):1869-1872.

14. Franklin H, Rajan M, Tseng CL, Pogach L, Sinha A, Mph M. Cost of lower-limb amputation in U.S. veterans with diabetes using health services data in fiscal years 2004 and 2010. J Rehabil Res Dev. 2014;51(8):1325-1330.

15. Wang C, Mai L, Yang C, Liu D, Sun K, Song W, Luo B, et al. Reducing major lower extremity amputations after the introduction of a multidisciplinary team in patient with diabetes foot ulcer. BMC Endocr Disord. 2016;16(1):38.

16. Oliver TI, Mutluoglu M. Diabetic foot ulcer. In: StatPearls. Treasure Island (FL), 2019.

17. Everett E, Mathioudakis N. Update on management of diabetic foot ulcers. Ann N Y Acad Sci. 2018;1411(1):153165.

18. Blume $\mathrm{P}, \mathrm{Wu} \mathrm{S}$. Updating the diabetic foot treatment algorithm: recommendations on treatment using advanced medicine and therapies. Wounds. 2018;30(2):29-35.

19. Quilici MT, Del Fiol Fde S, Vieira AE, Toledo MI. Risk factors for foot amputation in patients hospitalized for diabetic foot infection. J Diabetes Res. 2016;2016:8931508.

20. Armstrong DG, Fisher TK, Lepow B, White ML, Mills JL. Pathophysiology and principles of management of the diabetic foot. In: Fitridge R, Thompson M, eds. Mechanisms of vascular disease: a reference book for vascular specialists. Adelaide. 2011.

21. Aalaa M, Malazy OT, Sanjari M, Peimani M, MohajeriTehrani M. Nurses' role in diabetic foot prevention and care; a review. J Diabetes Metab Disord. 2012;11(1):24.

22. Georgescu DE, Mustatea P, Mihalache O, Bobirca F, Agache A, Georgescu TF, Chiriac O, et al. Surgical Management of Diabetic Neuropathy Foot Complications. Chirurgia (Bucur). 2018;113(5):634-643.

23. Shin JY, Roh SG, Chang SC, Lee NH. Can we predict vascular status and culture result based through wound status in diabetic foot infection? Medicine (Baltimore). 2019;98(12):e14892.

24. Shatnawi NJ, Al-Zoubi NA, Hawamdeh HM, Khader YS, Garaibeh K, Heis HA. Predictors of major lower limb amputation in type 2 diabetic patients referred for hospital care with diabetic foot syndrome. Diabetes Metab Syndr Obes. 2018;11:313-319.

25. Allie DE, Hebert CJ, Lirtzman MD, Wyatt CH, Keller VA, Khan MH, Khan MA, et al. Critical limb ischemia: a global epidemic.A critical analysis of current treatment unmasks the clinical and economic costs of CLI. EuroIntervention. 2005;1(1):75-84.

26. Goodney PP, Travis LL, Nallamothu BK, Holman K, Suckow B, Henke PK, Lucas FL, et al. Variation in the use of lower extremity vascular procedures for critical limb ischemia. Circ Cardiovasc Qual Outcomes. 2012;5(1):94102. 
27. Okonkwo UA, DiPietro LA. Diabetes and Wound Angiogenesis. Int J Mol Sci. 2017;18(7):1419.

28. Pendsey SP. Understanding diabetic foot. Int J Diabetes Dev Ctries. 2010;30(2):75-79.

29. Chilton RJ, Gallegos KM, Silva-Cardoso J, Oliveros $\mathrm{R}$, Pham S. The evolving role of the cardiologist in the management of type 2 diabetes. Curr Diab Rep. 2018;18(12):144.

30. Bruntz M, Berg C, Garcia J, Price C. A diabetic limb salvage program in an at-risk diabetic population: can it be successful? San Fransisco, CA: American Diabetes Association; 2014.

31. Pedrosa HC, Leme LAP, Novaes C, Saigg M, Sena F, Gomes EB, Coutinho AM, et al. The diabetic foot in South America: progress with the Brazilian Save the Diabetic Foot project. Int Diabetes Monit. 2004;16(Neuropathy Issue):10-17.

32. Krishnan S, Nash F, Baker N, Fowler D, Rayman G. Reduction in diabetic amputations over 11 years in a defined U.K. population: benefits of multidisciplinary team work and continuous prospective audit. Diabetes Care. 2008;31(1):99-101.

33. Tseng CL, Helmer D, Rajan M, Tiwari A, Miller D, Crystal S, Safford M, et al. Evaluation of regional variation in total, major, and minor amputation rates in a national health-care system. Int J Qual Health Care. 2007;19(6):368-376.

34. Tseng CL, Rajan M, Miller DR, Hawley G, Crystal S, Xie M, Tiwari A, et al. Use of administrative data to risk adjust amputation rates in a national cohort of medicare-enrolled veterans with diabetes. Med Care. 2005;43(1):8892.

35. Sambamoorthi U, Tseng CL, Rajan M, Anjali T, Findley PA, Pogach L. Initial nontraumatic lower-extremity amputations among veterans with diabetes. Med Care. 2006;44(8):779-787.

36. Tseng CL, Rajan M, Miller DR, Lafrance JP, Pogach L. Trends in initial lower extremity amputation rates among Veterans Health Administration health care System users from 2000 to 2004. Diabetes Care. 2011;34(5):11571163.

37. Pogach L, Charns MP, Wrobel JS, Robbins JM, Bonacker KM, Haas L, Reiber GE. Impact of policies and performance measurement on development of organizational coordinating strategies for chronic care delivery. Am J Manag Care. 2004;10(2 Pt 2):171-180.

38. Frye RL. President's page: role of the cardiologist in peripheral vascular disease. J Am Coll Cardiol. 1991;18(2):641-642.

39. Creager MA, Gornik HL, Gray BH, Hamburg NM, Iobst WF, Mohler ER, 3rd, White CJ. COCATS 4 task force 9: training in vascular medicine. J Am Coll Cardiol. 2015;65(17):1832-1843.

40. Cameron SJ. Vascular medicine: the eye cannot see what the mind does not know. J Am Coll Cardiol. 2015;65(25):2760-2763.

41. Goodney PP, Beck AW, Nagle J, Welch HG, Zwolak RM. National trends in lower extremity bypass surgery, endovascular interventions, and major amputations. J Vasc Surg. 2009;50(1):54-60.

42. Aronson D, Edelman ER. Coronary artery disease and diabetes mellitus. Cardiol Clin. 2014;32(3):439-455.

43. Cardiovascular Diseases (CVDs), 2017. Available from: https://www.who.int/en/news-room/fact-sheets/detail/ cardiovascular-diseases-(cvds).

44. Bhatt DL, Eagle KA, Ohman EM, Hirsch AT, Goto S, Mahoney EM, Wilson PW, et al. Comparative determinants of 4-year cardiovascular event rates in stable outpatients at risk of or with atherothrombosis. JAMA. 2010;304(12):1350-1357.

45. Nicosia A, Cernetti C, Cremonesi A, Goktekin O, Sauguet A, Wijns W, Musumeci G. PCR Peripheral @ GISE: a unique educational link between peripheral endovascular solutions and interventional cardiologists. EuroIntervention. 2017;13(9):e1013-e1017.

46. Jones WS, Patel MR. Antithrombotic Therapy in Peripheral Artery Disease: Generating and Translating Evidence Into Practice. J Am Coll Cardiol. 2018;71(3):352-362.

47. Khanolkar UB, Ephrem B. Endovascular reconstruction of popliteal and infrapopliteal arteries for limb salvage and wound healing in patients with critical limb ischemia - A retrospective analysis. Indian Heart J. 2016;68(1):7782.

48. Powell R, Menard M, Farber A, Rosenfield K, Goodney $\mathrm{P}$, Gray B, Lookstein R, et al. Comparison of specialties participating in the BEST-CLI trial to specialists treating peripheral arterial disease nationally. J Vasc Surg. 2019;69(5):1505-1509. 